\title{
The thyroid hormone levels and glucose and lipid metabolism in children with type 1 diabetes: a correlation analysis
}

\author{
Chuanjie Yuan ${ }^{1,2}$, Xiaomei Sun ${ }^{1,2}$, Yin $\mathrm{Liu}^{1,2}, \mathrm{Jin}^{\mathrm{Wu}}{ }^{1,2}$ \\ ${ }^{1}$ Department of Pediatric Endocrinology and Metabolism, West China Second Hospital of Sichuan University, Chengdu, China; ${ }^{2}$ Key Laboratory of \\ Birth Defects and Related Diseases of Women and Children, Sichuan University, Ministry of Education, Chengdu, China \\ Contributions: (I) Conception and design: C Yuan, J Wu; (II) Administrative support: All authors; (III) Provision of study materials or patients: C Yuan, \\ X Sun; (IV) Collection and assembly of data: C Yuan, J Wu; (V) Data analysis and interpretation: X Sun, Y Liu; (VI) Manuscript writing: All authors; \\ (VII) Final approval of manuscript: All authors. \\ Correspondence to: Jin Wu. No. 20, Section 3, Renmin South Road, Wuhou District, Chengdu 610000, China. Email: ujwure@163.com.
}

Background: Type 1 diabetes mellitus (T1DM) is common in clinical setting, the relationship between thyroid hormone levels and glucose and lipid metabolism in patients with T1DM remains unclear. We attempted to analyze the correlation of thyroid hormone levels and blood glucose and lipid metabolism in children with normal thyroid function.

Methods: Children with T1DM were selected, and 85 healthy children who underwent physical examinations in our hospital as control group. The characteristics and laboratory testing results were compared, the correlation of thyroid hormone levels with blood glucose and blood lipids was analyzed by Pearson correlation analysis.

Results: A total of 167 participants were included. the low-density lipoprotein-cholesterol (LDL-C), triglyceride (TG), fasting blood glucose (FBG), and glycated hemoglobin (HbAlc) in T1DM patients were higher than those in healthy controls, while the high-density lipoprotein-cholesterol (HDL-C), thyroid stimulating hormone (TSH) and free triiodothyronine (FT3) in T1DM patients were lower than that of healthy children (all $\mathrm{P}<0.05)$. TSH was positively correlated with LDL-C $(\mathrm{r}=0.169, \mathrm{P}=0.032)$, TC $(\mathrm{r}=0.182$, $\mathrm{P}=0.017)$ and TG $(\mathrm{r}=0.197, \mathrm{P}=0.008)$, negatively correlated with FBG $(\mathrm{r}=-0.196, \mathrm{P}=0.023)$ and HbAlc $(\mathrm{r}=-0.328, \mathrm{P}=0.002)$. FT3 was negatively correlated with TG $(\mathrm{r}=-0.182, \mathrm{P}=0.011), \mathrm{FBG}(\mathrm{r}=-0.184, \mathrm{P}=0.009)$ and HbAlc ( $\mathrm{r}=-0.223, \mathrm{P}=0.005)$. TG in the high TSH group and the middle TSH group is higher than that of low TSH group. However, FBG and HbAlc were lower than those in the low TSH group (all $\mathrm{P}<0.05$ ). Compared with the low FT3 group, the TG, FBG and HbAlc decreased in the high and middle FT3 group (all $\mathrm{P}<0.05)$.

Conclusions: The serum TSH are closely associated with FT3 and glycolipid metabolism in children with T1DM, which may be the useful indicators to assess the severity of T1DM in clinical settings to provide insights into the management of T1DM.

Keywords: Type 1 diabetes mellitus (T1DM); thyroid; glucose; lipid; children; correlation

Submitted Jul 22, 2020. Accepted for publication Dec 18, 2020.

doi: 10.21037/tp-20-204

View this article at: http://dx.doi.org/10.21037/tp-20-204

\section{Introduction}

The incidence of type 1 diabetes mellitus (T1DM) is increasing globally (1). T1DM mostly occurs in adolescents, with regards to the relatively low incidence and relatively young patients, the T1DM do not get enough attention (2). The mortality and the incidence of related complications are high in patients with of T1DM (3). At present, the pathogenesis of T1DM has not been fully clarified. In recent years, more and more scholars have paid attention to 
the topic of T1DM, and the research on the pathogenesis of T1DM is gradually deepening.

Diabetes and thyroid diseases are the two most common diseases in the department of endocrinology, and the two diseases are closely related (4). Studies $(5,6)$ have shown that patients with T1DM or type 2 diabetes mellitus (T2DM) may have abnormal thyroid function. In addition to promote the growth and development of tissue, thyroid hormones can also affect the metabolism of sugar, fat and protein (7), and it's been reported that it participates in the development of T2DM $(8,9)$. However, there are very few studies on the relationship between thyroid hormone levels and glucose and lipid metabolism, especially in T1DM children with normal thyroid function.

Therefore, we aimed to investigate the thyroid hormone levels and blood glucose and lipid metabolism in children with normal thyroid function, to analyze the underlying correlation of abnormal blood glucose and lipids levels in children with T1DM, thereby to provide a reliable theoretical basis for the treatment of T1DM.

We present the following article in accordance with the STROBE reporting checklist (available at http://dx.doi. org/10.21037/tp-20-204).

\section{Methods}

\section{Ethical consideration}

Our study is a retrospective analysis. And the study conformed to the provisions of the Declaration of Helsinki (as revised in 2013) (10). This study has been certified and approved by the ethical committee of our hospital (20180933), and the written informed consents have been obtained from all the included patients.

\section{Patients}

Children with T1DM who were firstly diagnosed in our hospital from January 2019 to January 2020 were selected. The inclusion criteria were: (I) the age was 6-13 years old; (II) the diagnostic criteria of T1DM complied with relevant guidelines and consensus $(11,12)$; (III) patients were willing to participant in this study. The exclusion criteria were: (I) patients had acute complications of diabetes such as diabetic ketoacidosis; (II) the patients had abnormal thyroid function, that is, the electrochemiluminescence immunoassay method was used in accordance with the laboratory testing standards of our hospital. Patients with any abnormalities out of following ranges were excluded: free triiodothyronine (FT3) 2.8-7.1 pmol/L, free thyroxine (FT4) $12-22 \mathrm{pmol} / \mathrm{L}$, thyroid stimulating hormone (TSH) $0.27-4.20 \mu \mathrm{IU} / \mathrm{mL}$, thyroglobulin antibody $0-115.0 \mathrm{IU} / \mathrm{mL}$, thyroid peroxidase antibody $0-34.0 \mathrm{IU} / \mathrm{mL}$, thyroid stimulating hormone receptor antibody 0-1.750 IU/L; (III) the patient also suffered from other endocrine system diseases such as dwarfism and Cushing's syndrome; (IV) the patient were taking drugs that may affect the level of thyroid hormones, such as iodine, glucocorticoids, and amidoketone; (V) the patients did not agree to participant in this study.

In addition, we selected 85 healthy children who underwent physical examinations in our hospital as a normal control group. The inclusion criteria were: (I) the age was 6-13 years old; (II) the results of physical examinations were normal; (III) the children were well informed and agree to participant in this study. The children who did not agree to participant in this study were excluded.

\section{Data collection}

Endocrine professionals measured height, weight and blood pressure according to international standards. All subjects were fasting for more than $12 \mathrm{~h}$, and then venous blood was taken for clinical analysis the next morning. Automatic biochemical analyzer was used to measure routine biochemical indexes such as blood lipids, blood sugar, etc., electrochemiluminescence immunoassay was used to detect FT3, FT4 and TSH levels, and high-performance liquid chromatography (Olympus AU2900, Japan) was used to determine the level of HbA1c.

\section{Statistical analysis}

In this study, SPSS 23.0 statistical software was used for statistical data processing, and the results were expressed as mean \pm standard deviation. The comparison between the two groups was performed by independent sample $t$ test. And one-way analysis of variance (ANVOA) was used for comparison between the three groups, and the least significant difference (LSD) method was used for pairwise comparison of variance analysis. The correlation of thyroid hormone levels with blood glucose and blood lipids was analyzed by Pearson correlation analysis. In this study, $\mathrm{P}<0.05$ was considered as being statistically different.

\section{Results}

A total of 167 participants were included, specifically with 
Table 1 The characteristics of included patients

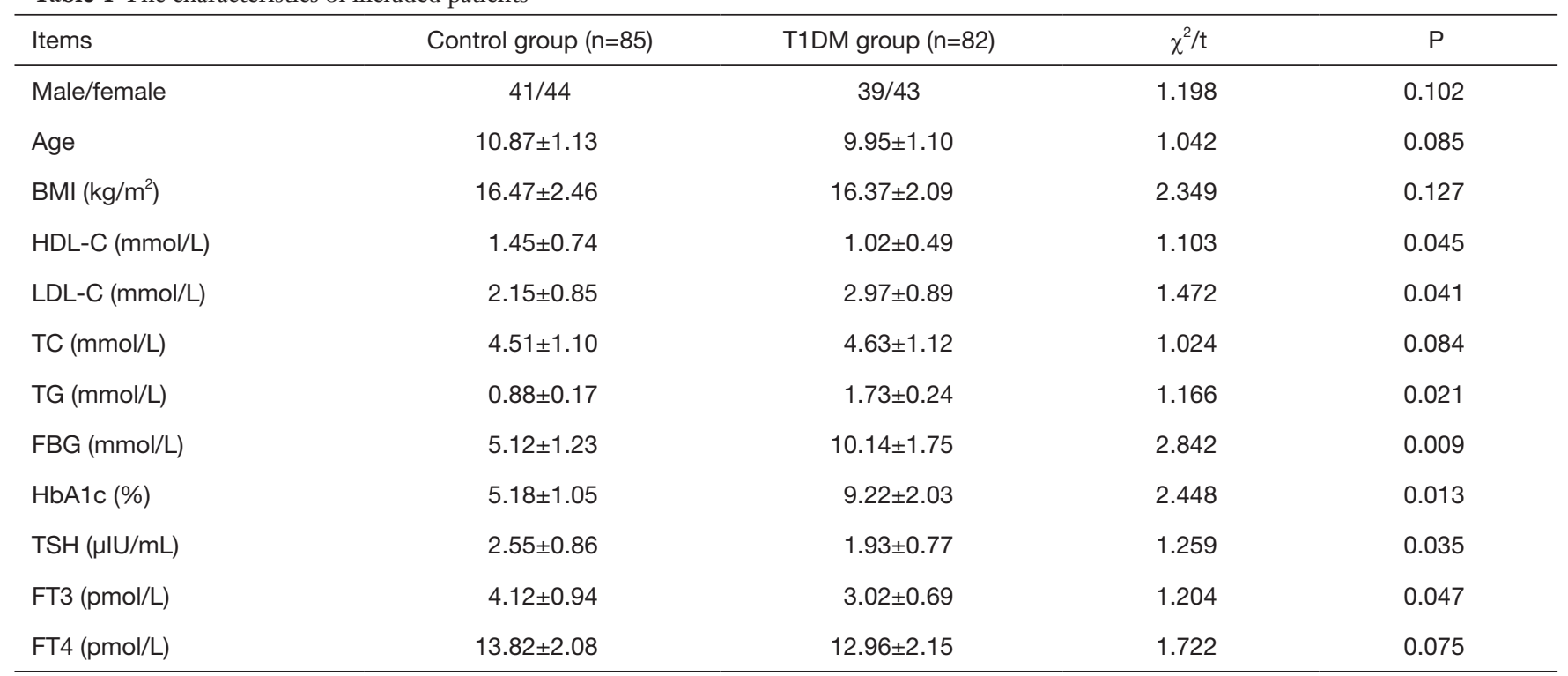

T1DM, type 1 diabetes mellitus; BMI, body mass index; HDL-C, high-density lipoprotein-cholesterol; LDL-C, low-density lipoprotein-cholesterol; TG, triglyceride; FBG, fasting blood glucose; HbAlc, glycated hemoglobin; TSH, thyroid stimulating hormone; FT3, free triiodothyronine; FT4, free thyroxine.

Table 2 Correlation analysis of thyroid hormone levels with blood glucose and blood lipids in patients with T1DM

\begin{tabular}{|c|c|c|c|c|c|c|}
\hline Items & \multicolumn{2}{|c|}{ TSH } & \multicolumn{2}{|c|}{ FT3 } & \multicolumn{2}{|c|}{ FT4 } \\
\hline HDL-C (mmol/L) & -0.063 & 0.242 & 0.095 & 0.124 & 0.087 & 0.201 \\
\hline LDL-C (mmol/L) & 0.169 & 0.032 & -0.098 & 0.069 & 0.115 & 0.093 \\
\hline TC (mmol/L) & 0.182 & 0.017 & -0.124 & 0.086 & -0.028 & 0.205 \\
\hline FBG (mmol/L) & -0.196 & 0.023 & -0.184 & 0.009 & 0.977 & 0.067 \\
\hline $\mathrm{HbA1c}(\%)$ & -0.328 & 0.002 & -0.223 & 0.005 & 0.182 & 0.106 \\
\hline
\end{tabular}

T1DM, type 1 diabetes mellitus; HDL-C, high-density lipoprotein-cholesterol; LDL-C, low-density lipoprotein-cholesterol; TG, triglyceride; FBG, fasting blood glucose; HbAlc, glycated hemoglobin; TSH, thyroid stimulating hormone; FT3, free triiodothyronine; FT4, free thyroxine.

85 healthy controls and 82 T1DM patients. As Table 1 presented, the low-density lipoprotein-cholesterol (LDL-C), triglyceride (TG), fasting blood glucose (FBG), and HbAlc in T1DM patients were higher than that of healthy controls, while the high-density lipoprotein-cholesterol (HDL-C), TSH and FT3 T1DM patients were lower than that of healthy children, and the difference was statistically significant (all $\mathrm{P}<0.05$ ). The gender, age, body mass index (BMI), total cholesterol (TC) and FT4 between the two groups were not significantly different (all $\mathrm{P}>0.05$ ).

\section{Correlation of thyroid hormone levels with blood glucose and blood lipids in patients with T1DM}

As Table 2 presented, Pearson correlation analysis indicated that in children with T1DM, TSH was positively correlated with LDL-C ( $\mathrm{r}=0.169, \mathrm{P}=0.032)$, TC $(\mathrm{r}=0.182, \mathrm{P}=0.017)$ and TG ( $\mathrm{r}=0.197, \mathrm{P}=0.008)$, negatively correlated with FBG $(\mathrm{r}=-0.196, \mathrm{P}=0.023)$ and HbAlc $(\mathrm{r}=-0.328, \mathrm{P}=0.002)$, but not significantly related to HDL-C. Besides, FT3 was negatively correlated with TG $(r=-0.182, P=0.011)$, FBG $(r=-0.184$, 
Table 3 Comparison of blood glucose and blood lipids grouped by tertile of thyroid hormone levels in T1DM patients

\begin{tabular}{|c|c|c|c|c|c|c|c|c|c|}
\hline Items & \multicolumn{3}{|c|}{$\mathrm{TSH}(\mu \mathrm{IU} / \mathrm{mP})$} & \multicolumn{3}{|c|}{ FT3 (pmol/L) } & \multicolumn{3}{|c|}{ FT4 (pmol/L) } \\
\hline $\mathrm{HDL}-\mathrm{C}(\mathrm{mmol} / \mathrm{L})$ & $1.18 \pm 0.31$ & $1.16 \pm 0.29$ & $1.09 \pm 0.17$ & $1.01 \pm 0.21$ & $1.12 \pm 0.27$ & $1.18 \pm 0.29$ & $1.12 \pm 0.31$ & $1.14 \pm 0.48$ & $1.19 \pm 0.82$ \\
\hline LDL-C (mmol/L) & $2.68 \pm 1.01$ & $2.71 \pm 1.08$ & $2.79 \pm 1.15$ & $2.73 \pm 0.69$ & $2.76 \pm 0.71$ & $2.79 \pm 0.77$ & $2.60 \pm 1.02$ & $2.71 \pm 1.15$ & $2.77 \pm 1.09$ \\
\hline $\mathrm{TC}(\mathrm{mmol} / \mathrm{L})$ & $4.62 \pm 1.22$ & $4.65 \pm 1.28$ & $4.69 \pm 1.17$ & $4.62 \pm 1.12$ & $4.68 \pm 1.27$ & $4.99 \pm 1.30$ & $4.97 \pm 1.26$ & $4.59 \pm 1.17$ & $4.33 \pm 1.02$ \\
\hline FBG (mmol/L) & $12.53 \pm 4.12$ & $10.28 \pm 3.19^{\star}$ & $8.16 \pm 2.15^{\star}$ & $11.73 \pm 2.97$ & $9.97 \pm 1.56^{*}$ & $9.73 \pm 1.44^{*}$ & $10.93 \pm 2.26$ & $10.43 \pm 2.14$ & $10.22 \pm 1.96$ \\
\hline HbA1c (\%) & $11.45 \pm 2.97$ & $9.98 \pm 2.01^{*}$ & $8.17 \pm 1.74^{\star \#}$ & $11.35 \pm 2.10$ & $9.36 \pm 1.95^{\star}$ & $9.31 \pm 1.97^{*}$ & $9.89 \pm 1.21$ & $9.92 \pm 1.37$ & $9.97 \pm 1.32$ \\
\hline
\end{tabular}

*, $\mathrm{P}<0.05$ compared with Tertile 1; ", $\mathrm{P}<0.05$ compared with Tertile 2. T1DM, type 1 diabetes mellitus; HDL-C, high-density lipoprotein-cholesterol; LDL-C, low-density lipoprotein-cholesterol; TG, triglyceride; FBG, fasting blood glucose; HbAlc, glycated hemoglobin; TSH, thyroid stimulating hormone; FT3, free triiodothyronine; FT4, free thyroxine.

$\mathrm{P}=0.009)$ and HbAlc $(\mathrm{r}=-0.223, \mathrm{P}=0.005)$, no correlations between FT3 and HDL-C, LDL-C, TC were found (all $\mathrm{P}<0.05)$. and no correlations between FT4 and HDL-C, LDL-C, TC, TG, FBG and HbA1c were found (all $\mathrm{P}<0.05$ ).

\section{Comparison of blood glucose and blood lipids grouped by tertile of thyroid hormone levels in T1DM patients}

Children with T1DM were divided into 3 groups according to TSH tertiles. As Table 3 presented, the results showed that the TG in the high TSH group and the middle TSH group is higher than that of low TSH group. However, FBG and HbAlc were lower than those in the low TSH group, and the differences were statistically significant (all $\mathrm{P}<0.05)$. There were no significant differences in HDL-C, LDL-C, and TC among three groups (all $\mathrm{P}>0.05$ ). Besides, according to the FT3 tertile, T1DM children were divided into three groups, the results indicated that compared with the low FT3 group, the TG, FBG and HbAlc decreased in the high and middle FT3 group, and the differences were statistically significant (all $\mathrm{P}<0.05$ ). There weren't significant differences between FT3 and HDL-C, LDL-C and TC (all $\mathrm{P}>0.05$ ). The children with TIDM were divided into 3 groups according to the FT4 tertile, and the results indicated that there were no significant differences in HDL-C, LDL-C, TC, TG, FBG and HbAlc in each group (all $\mathrm{P}>0.05$ ).

\section{Discussion}

The T1DM in children is caused by absolute lack of insulin. Due to its poor pancreatic islet function and large fluctuations in blood sugar, it is more likely that T2DM to be complicated by diabetic ketoacidosis, severe hypoglycemia, diabetic retinopathy, diabetic nephropathy and other complications $(13,14)$, it may seriously affect children's quality of life and cause financial burdens on families (15). Good glycemic control helps to delay or prevent complications (16). T1DM patients are often accompanied by dyslipidemia, and dyslipidemia is an important risk factor for cardiovascular disease (17). Therefore, evaluating the risk factors that affect glucose and lipid metabolism disorders in TIDM patients is especially important.

In addition to promoting tissue differentiation, maintaining the excitability of the nervous system and promoting protein synthesis (18), thyroid hormones can also affect sugar and lipid metabolism (19), and it participates in the development of various diseases such as diabetes, coronary heart disease (20). Several reports (21-23) have explored the relationship between thyroid hormones and glucose and lipid metabolism in T2DM patients, and have provide some theoretical basis for the treatment and T2DM. it's been found in T2DM female patients with normal thyroid function, TSH is positively correlated with TC and LDL-C, but it has no significant correlation with TG and HDL-C, but in male patients, TSH and TC, TG, LDL-C and HDL-C have no obvious correlation (24). Besides, it has found that in T2DM patients with normal thyroid function, TSH was positively correlated with TG and HbAlc (25), and it has no significant correlation with FBG, LDL-C and HDL-C, while FT3 and FT4 had no significant relations to glucose and lipid metabolism (26). Previous studies (27-29) have indicated that in T2DM patients, thyroid hormone levels are closely related 
to glucose and lipid metabolism, but there are few studies on the relationship between thyroid hormone levels and glucose and lipid metabolism in T1DM patients, especially in TIDM children with normal thyroid function. We have explored the relationship between thyroid hormone levels and blood glucose and blood lipid metabolism in children with normal thyroid function and primary T1DM. The results indicate that TSH is positively correlated with LDL-C, TC and TG, negatively correlated with FBG and HbAlc, and FT3 is negatively correlated with TG, FBG and HbAlc.

When patients with T1DM are in a state of hyperglycemia, insulin secretion is relatively insufficient, and the body's glucose metabolism activity is reduced, the energy utilization of thyroid follicular cells is reduced, which causes iodine pump dysfunction and the thyroid's response to $\mathrm{TSH}$ is reduced $(30,31)$. It reduces the thyroid hormone synthesis, which in turn affects the activity of 5 '-deiodinase, and FT3 levels are reduced (32). When blood glucose is poorly controlled for a long time, the body is in an obvious metabolic disorder, which may indirectly affect the function of the hypothalamic pituitary thyroid axis system (33). Insensitivity leads to a decrease in TSH secretion. FT3 affects the antilipolytic effect of insulin, resulting in a significant increase in free FFA concentration and an increase in TG synthesis (34). In addition, when TSH increases, although the synthesis of cholesterol decreases, the rate of excretion is lower, which increases the concentration of LDL-C, TC and TG in the blood $(35,36)$, but the specific mechanism needs to be further studied.

The current study found that diabetes combined with abnormal thyroid hormone is mainly manifested by the reduction of TSH and/or T3 levels, and the changes of T4 are mostly insignificant. This study also found in patients with T1DM, compared with the healthy control group, TSH and FT3 are reduced, and FT4 has no obvious changes. Some scholars (37-39) believe that this phenomenon is a state of low metabolism in the body. To avoid consumption caused by excessive metabolism, a regulation or defense mechanism may link, further researches on the specific mechanism is still needed. The current study has found that diabetes combined with abnormal thyroid hormone is mainly manifested by the reduction of TSH and/or T3 levels. This study also found in patients with T1DM, compared with the healthy control group, TSH and FT3 are reduced, and FT4 has no obvious changes. Some researches on blood lipids and type 1 diabetes have made some progress, but there is no unified conclusion on the relations. Further researches are needed to identify the molecular biology and pathophysiological mechanisms.

\section{Conclusions}

In conclusion, we have found that in T1DM patients with normal thyroid function, thyroid hormone levels are closely related to glucose and lipid metabolism, so it is particularly important to detect thyroid function in T1DM patients, which is helpful for early detection and correction of abnormal thyroid function, and it may further improve the disorder of glucose and lipid metabolism and prevent the complications of T1DM. Still, the immunity system disorder is reported to be associated with T1DM, testing the lymphocytes and inflammatory factors may provide more elucidations on the mechanisms of T1DM, which should be further evaluated in future studies.

\section{Acknowledgments}

Funding: None.

\section{Footnote}

Reporting Checklist: The authors have completed the STROBE reporting checklist. Available at http://dx.doi. org/10.21037/tp-20-204

Data Sharing Statement: Available at http://dx.doi. org/10.21037/tp-20-204

Conflicts of Interest: All authors have completed the ICMJE uniform disclosure form (available at http://dx.doi. org/10.21037/tp-20-204). The authors have no conflicts of interest to declare.

Ethical Statement: The authors are accountable for all aspects of the work in ensuring that questions related to the accuracy or integrity of any part of the work are appropriately investigated and resolved. This study has been certified and approved by the ethical committee of our hospital (20180933), and the written informed consents have been obtained from all the included patients. The study was conducted in accordance with the Declaration of Helsinki (as revised in 2013).

Open Access Statement: This is an Open Access article distributed in accordance with the Creative Commons Attribution-NonCommercial-NoDerivs 4.0 International 
License (CC BY-NC-ND 4.0), which permits the noncommercial replication and distribution of the article with the strict proviso that no changes or edits are made and the original work is properly cited (including links to both the formal publication through the relevant DOI and the license). See: https://creativecommons.org/licenses/by-nc-nd/4.0/.

\section{References}

1. Janež A, Guja C, Mitrakou A, et al. Insulin Therapy in Adults with Type 1 Diabetes Mellitus: a Narrative Review. Diabetes Ther 2020;11:387-409.

2. Sirakaya E, Kucuk B, Sirakaya HA. The Influence of Type 1 Diabetes Mellitus on Amplitude of Accommodation. Curr Eye Res 2020;45:873-8.

3. Desai S, Deshmukh A. Mapping of Type 1 Diabetes Mellitus. Curr Diabetes Rev 2020;16:438-41.

4. Cieluch A, Uruska A, Zozulinska-Ziolkiewicz D. Can We Prevent Mitochondrial Dysfunction and Diabetic Cardiomyopathy in Type 1 Diabetes Mellitus? Pathophysiology and Treatment Options. Int J Mol Sci 2020;21:2852.

5. Nevo-Shenker M, Phillip M, Nimri R, et al. Type 1 diabetes mellitus management in young children: implementation of current technologies. Pediatr Res 2020;87:624-9.

6. Castellanos L, Tuffaha M, Koren D, et al. Management of Diabetic Ketoacidosis in Children and Adolescents with Type 1 Diabetes Mellitus. Paediatr Drugs 2020.

7. Benvenga S, Pintaudi B, Vita R, et al. Serum thyroid hormone autoantibodies in type 1 diabetes mellitus. J Clin Endocrinol Metab 2015;100:1870-8.

8. Ferrari SM, Fallahi P, Galetta F, et al. Thyroid disorders induced by checkpoint inhibitors. Rev Endocr Metab Disord 2018;19:325-33.

9. Jonsdottir B, Larsson C, Carlsson A, et al. Thyroid and Islet Autoantibodies Predict Autoimmune Thyroid Disease at Type 1 Diabetes Diagnosis. J Clin Endocrinol Metab 2017;102:1277-85.

10. World Medical A. World Medical Association Declaration of Helsinki: ethical principles for medical research involving human subjects. JAMA 2013;310:2191-4.

11. Franz MJ, MacLeod J, Evert A, et al. Academy of Nutrition and Dietetics Nutrition Practice Guideline for Type 1 and Type 2 Diabetes in Adults: Systematic Review of Evidence for Medical Nutrition Therapy Effectiveness and Recommendations for Integration into the Nutrition Care Process. J Acad Nutr Diet 2017;117:1659-79.
12. American Diabetes A. (2) Classification and diagnosis of diabetes. Diabetes Care 2015;38 Suppl:S8-S16.

13. Mendez CE, Umpierrez GE. Management of Type 1 Diabetes in the Hospital Setting. Curr Diab Rep 2017;17:98.

14. Chiang JL, Maahs DM, Garvey KC, et al. Type 1 Diabetes in Children and Adolescents: A Position Statement by the American Diabetes Association. Diabetes Care 2018;41:2026-44.

15. McKnight JA, Wild SH, Lamb MJ, et al. Glycaemic control of Type 1 diabetes in clinical practice early in the 21st century: an international comparison. Diabet Med 2015;32:1036-50.

16. Reddy M, Rilstone S, Cooper P, et al. Type 1 diabetes in adults: supporting self management. BMJ 2016;352:i998.

17. Candler T, Mahmoud O, Edge J, et al. Hypercholesterolaemia screening in Type 1 diabetes: a difference of opinion. Diabet Med 2017;34:983-6.

18. Tost M, Monreal JA, Armario A, et al. Targeting Hormones for Improving Cognition in Major Mood Disorders and Schizophrenia: Thyroid Hormones and Prolactin. Clin Drug Investig 2020;40:1-14.

19. Tsibulnikov S, Maslov L, Voronkov N, et al. Thyroid hormones and the mechanisms of adaptation to cold. Hormones (Athens) 2020;19:329-39.

20. Colella M, Cuomo D, Giacco A, et al. Thyroid Hormones and Functional Ovarian Reserve: Systemic vs. Peripheral Dysfunctions. J Clin Med 2020;9:1679.

21. Gutch M, Rungta S, Kumar S, et al. Thyroid functions and serum lipid profile in metabolic syndrome. Biomed $\mathrm{J}$ 2017;40:147-53.

22. Guarnizo-Poma M, Paico-Palacios S, Pantoja-Torres B, et al. Association between free thyroid hormones values and the lipid profile in middle-aged women with chronic symptoms. Diabetes Metab Syndr 2018;12:531-5.

23. Zarei M, Zaeemi M, Rashidlamir A. Effects of testosterone enanthate treatment in conjunction with resistance training on thyroid hormones and lipid profile in male Wistar rats. Andrologia 2018;50. doi: 10.1111/and.12862.

24. Zhang Y, Lu P, Zhang L, et al. Association between lipids profile and thyroid parameters in euthyroid diabetic subjects: a cross-sectional study. BMC Endocr Disord 2015;15:12.

25. Jatwa R, Parmar HS, Panda S, et al. Amelioration of corticosteroid-induced type 2 diabetes mellitus by rosiglitazone is possibly mediated through stimulation of thyroid function and inhibition of tissue lipid peroxidation in mice. Basic Clin Pharmacol Toxicol 2007;101:177-80. 
26. Petrosyan L. Relationship between high normal TSH levels and metabolic syndrome components in type 2 diabetic subjects with euthyroidism. J Clin Transl Endocrinol 2015;2:110-3.

27. Delitala AP, Fanciulli G, Pes GM, et al. Thyroid Hormones, Metabolic Syndrome and Its Components. Endocr Metab Immune Disord Drug Targets 2017;17:56-62 .

28. Senese R, Lasala P, Leanza C, et al. New avenues for regulation of lipid metabolism by thyroid hormones and analogs. Front Physiol 2014;5:475.

29. Jang J, Kim Y, Shin J, et al. Association between thyroid hormones and the components of metabolic syndrome. BMC Endocr Disord 2018;18:29.

30. Ahi S, Amouzegar A, Gharibzadeh S, et al. Trend of lipid and thyroid function tests in adults without overt thyroid diseases: A cohort from Tehran thyroid study. PLoS One 2019;14:e0216389.

31. Karar T, Alhammad RI, Fattah MA, et al. Relation between glycosylated hemoglobin and lipid and thyroid hormone among patients with type 2 diabetes mellitus at King Abdulaziz Medical City, Riyadh. J Nat Sci Biol Med 2015;6:S75-9.

32. Finan B, Clemmensen C, Zhu Z, et al. Chemical Hybridization of Glucagon and Thyroid Hormone Optimizes Therapeutic Impact for Metabolic Disease. Cell 2016;167:843-57.e14.

Cite this article as: Yuan C, Sun X, Liu Y, Wu J. The thyroid hormone levels and glucose and lipid metabolism in children with type 1 diabetes: a correlation analysis. Transl Pediatr 2021;10(2):276-282. doi: 10.21037/tp-20-204
33. Amouzegar A, Kazemian E, Gharibzadeh S, et al. Association between thyroid hormones, thyroid antibodies and insulin resistance in euthyroid individuals: A population-based cohort. Diabetes Metab 2015;41:480-8.

34. Delitala AP, Delitala G, Sioni P, et al. Thyroid hormone analogs for the treatment of dyslipidemia: past, present, and future. Curr Med Res Opin 2017;33:1985-93.

35. Zhu X, Cheng SY. New insights into regulation of lipid metabolism by thyroid hormone. Curr Opin Endocrinol Diabetes Obes 2010;17:408-13.

36. Szkudelski T, Michalski W, Szkudelska K. The effect of thyroid hormones on blood insulin level and metabolic parameters in diabetic rats. J Physiol Biochem 2003;59:71-6.

37. Jayanthi R, Srinivasan AR, Gopal N, et al. Association of divalent cations and insulin resistance with thyroid hormones in patients with type 2 diabetes mellitus. Diabetes Metab Syndr 2017;11 Suppl 2:S885-90.

38. Bulum T, Kolaric B, Duvnjak L. Insulin sensitivity modifies the relationship between thyroid function and lipid profile in euthyroid type 1 diabetic patients. Endocrine 2012;42:139-45.

39. Wolide AD, Zawdie B, Alemayehu T, et al. Association between thyroid hormone parameters and dyslipidemia among type 2 diabetes mellitus patients: Comparative cross-sectional study. Diabetes Metab Syndr 2017;11 Suppl 1:S257-62. 